IJBPAS, November, 2019, 8(11): 2128-2139

ISSN: 2277-4998

International Journal of Biology, Pharmacy

and Allied Seiences (IJBPAS)

'A Bridge Betusen Caboratory and Qender'

Www.iibpas.com

SIGNIFICANCE OF PREDICTIVE VARIABLES OF MEDICAL IMPORTANCE AND

THEIR DIFFERENTIAL EXPRESSION IN PREGNANT FEMALES: STUDY OF FUTURE PERINATAL OUTCOMES

\title{
TARIQ $\mathbf{N}^{3}$, ANWER $\mathrm{K}^{2}$, QURESHI ZH ${ }^{4}$, ZAHID A ${ }^{1}$, WAQUAR $\mathbf{S}^{1}$ AND MALIK $\mathrm{A}^{{ }^{*}}$
}

1: Institute of Molecular biology and biotechnology (IMBB), The University of Lahore-Pakistan

2: Biochemistry department, Sheikh Zayed Medical College, Rahim Yar Khan-Pakistan

3: M.Islam Medical and Dental College, Gujranwala-Pakistan

4: Multan Medical and Dental College-Multan-Pakistan

\begin{tabular}{|c|}
\hline${ }^{*}$ Corresponding Author: Dr. Arif Malik (PhD): E Mail: $\underline{\text { arifuaf@yahoo.com }}$ \\
\hline Received $8^{\text {th }}$ March 2019; Revised 11 \\
\hline$\underline{\text { https://doi.org/10.31032/IJBPAS/2019/8.11.4859 }}$ \\
\hline
\end{tabular}

ABSTRACT

Normal pregnancy is associated with oxidative stress injury due to high energy demand of foetus during whole pregnancy and labour. Excessive oxygen demand leads to free radical generation and poor perinatal outcomes in most of the cases. Oxidative stress is responsible for a variety of degenerative processes in new-born babies in future. Measurement of oxidatively modified DNA, Lipids peroxidation products in biological samples may be helpful or will be served as predispositional important variables for future perinatal outcomes. Current study was designed to assess the differential expressions of malondialdehyde, isoprostanes and 8-Hydroxyguanosine in cord blood, serum and saliva immediately after labour in normal pregnancy. Thirty normal pregnant females and thirty clinically apparently healthy non-pregnant females aging from 25-35 years were recruited. Cord blood and saliva samples were collected and MDA, Isoprostanes and $8 \mathrm{OHdG}$ levels evaluated. Data was analyzed by performing Independent t-test through SPSS version 16.It was found that the malondialdehyde (MDA) levels raised significantly $(p=0.038)$ in cord blood serum $(1.43 \pm 0.0048$ Vs $1.14 \pm 0.0027 \mathrm{nmoles} / \mathrm{ml})$, saliva $(0.48 \pm 0.0020$ Vs $0.031 \pm 0.0019 \mathrm{nmoles} / \mathrm{ml})$ and cord blood erythrocytes $(0.34 \pm 0.0011$ Vs $0.02 \pm 0.007 \mathrm{nmoles} / \mathrm{ml})$ 
from the normal pregnant females as compared to healthy non-pregnant females. Likewise the levels of isoprostanes and 8-hydroxy guanosine differed significantly $(\mathrm{p}=0.021)$ and $(\mathrm{p}=0.006)$ between subject and controls respectively. Current study suggest that due to increased lipidperoxidation the levels of their biomarkers also increased. Sothese markers have significant diagnostic value and predispositional variables for early detection of oxidative stress generated during pregnancy and used for future perinatal outcomes to assess the suspected complications.

Keywords: MDA (Malondialdehyde), isoprostanes (IsP)), 8-OHdG (8-hydroxygunosine), Perinatal INTRODUCTION

Umbilical cord develops from the connecting stalk, is $50 \mathrm{~cm}$ in length and $2 \mathrm{~cm}$ in diameter and made up of mesodermal connective tissue whose function is to protect the blood vessels $[1,2]$. After the delivery of baby the umbilical cord is cut, some residual blood leftovers in the veins and arteries of the placenta this blood is known as placental blood or umbilical "cord blood". Cord blood has all the components of blood WBCs, RBCs, platelets and plasma. As in bone marrow which is a reservoir of blood forming stem cells, cord blood is also full of this source. So due to this reason cord blood can be used for new transplant in diseased patients alternative to bone marrow [3]. Till now more than 90 different types of diseases have been treated with unrelated cord blood transplants such as treatment for genetic diseases, blood malignancies, and immune deficiencies and used as regenerative therapies [4]. Blood in cord indicates the fetal and utero-placental position, it is distinguished by the maternal conditions like hypoxia, anemia, hypertension, inadequate size of placenta and it also involves fetal status of the patients. Moreover, the discussed venous blood of fetus is oxygenated and leads to the difficulties in the inherent to the fetus leading to anemia and CVD. If umbilical artery blood is academic, it naturally proposes that anaerobic metabolism happened. Anaerobic metabolism occurs when oxygen is not available, and is therefore an indicator that a hypoxic incident happened. Hypoxic ischemic procedures can result in pathological condition. Permanent damage to the brain may lead to the seizures in cases diagnosed with the hypoxic ischemic encephalopathy (HIE) [5].

Oxidative stress is an imbalance between the generation of highly reactive free radicals and ability of our body to counteract and detoxify their harmful effects through neutralization by antioxidants [6]. 
These oxidant or the reactive oxygen species are those reactive molecules which have unpaired one or more electrons in their outer molecular orbits and are in unstable form which requires electrons from neighboring molecules for their stabilization, the two main oxidant species are reactive oxygen species(ROS) and reactive nitrogen species (RNS) [7]. These reactive oxygen species exerts the oxidative damage to lipids, proteins and DNA individually or collectively ranging from signal transduction process failure to bio-molecular damage and cell death. Oxidative damage to lipids triggers the chain reaction of lipid peroxidation results in formation of various cytotoxic aldehydes like malondialdehyde (MDA) [8] and f2-isoprostanes as the intermediate products [9]. This is being measured widely to show evidence of oxidant (Free radical injury) in biological samples like saliva, urine and blood. In DNA these oxidant species damages nucleobases especially guanine and with the formation of 8-oxoguanine, which have many mutagenic and carcinogenic effects [10]. Antioxidant system which come in play to detoxify the effect of reactive oxygen species is maintained by key antioxidant enzymes e.g. superoxide dismutase, catalase, glutathione peroxides and thioredoxin reductase as well as non-enzymatic antioxidants such as vitamin $\mathrm{E}$, vitamin $\mathrm{C}$, and flavonoid [11].

In biological systems oxidative reactions have essential part, and have also bad effect on body when there is imbalance between oxidant and antioxidant defense system ,one of such example is pregnancy in which normal pregnancy is a physiological condition where an increase in free radicals generation due to high energy demand of body during pregnancy but when pregnancy is complicated with some gestational diseases such as pre-eclampsia and gestational diabetes, free radicals generation increases significantly, and results in more oxidative damage. We can correlate the oxidative stress biomarkers of mother and baby cord blood. Important oxidative stress markers are as following (carbonyl group, lipid peroxidation, DNA damage) [12]. Other biomarkers such as CRP (C-reactive protein), IL-6, IL-8, TNF are considered as important biomarker of neonatal sepsis [13]. Pregnancy is a commonly depressed physiological state and it has been associated with oxidative stress injury [14]. Oxidative stress in this state has been related with some problems e.g. preeclampsia, intrauterine growth retardation (IUGR), intra uterine fetal death (IUD) and perinatal death [15]. Pregnant ladies increase their weight appropriately as 
the pregnancy progresses and this is mainly due to the growth of the baby, inability of pregnant ladies to increase weight appropriately has been due to with an increase oxidative stress injury [16]. In pregnant females more generation of free radicals during whole gestation and labour occurs, these toxic radicals are overcome by the antioxidant defence system, the maturation will come through out gestation step by step. A mature and healthy infant have more capability to tolerate these changes in oxygen concentration during gestation and at the time of delivery from low to high concentration. There will be problem when baby in utero cannot reach their maximum maturity, and suffer premature delivery or intrauterine growth retardation (IUGR) and obviously there will be imbalance in oxidant and antioxidant system and baby have low capability to overcome this problem so due to prematurity baby suffer such complications e.g., respiratory distress syndrome (RDS), necrotizing enterocolitis (NEC), chronic lung disease, retinopathy of prematurity and intraventricular hemorrhage (IVH) [17]. Thus the fetuses born with hypoxia, IUGR, prematurity or any other disease related to mother or fetus, in both condition fetus have excessive generation of free radicals and low potency to detoxify them [18]. Oxidative stress have been implicated in many human diseases such as diabetes, alcoholic liver disease, ARDS, Alzheimer disease and retinopathy of prematurity, the main targets of free radicals are DNA, protein, lipid peroxidation, and their relative most important and non-invasive biomarkers are 8, hydroxyguanosine, f2 isoprostanes and MDA [19]. All of these are very reliable and most important biomarkers and easily approachable in biological samples like blood, urine and saliva, these biomarkers are of oxidative stress, and their high level in plasma, urine and saliva indicates underlying disease like diabetes, ARDS or imbalance between oxidants and antioxidants [20]. Present study was designed to evaluate the differential expression of isoprostanes, malondialdehyde, 8-hydroxyguanosine and their specificity in different medias.

\section{MATERIALS AND METHODS}

The present study was designed to investigate the key metabolites or predispositional variables during labor in normal pregnancy which may be helpful in future perinatal outcomes. All the subjects (Normal pregnant females) were screened at the department of obstetrics gynaecology, DHQ hospital D.G. khan. Thirty normal pregnant females were in the age group of 
25-35 years were eligible for inclusion in the study. Informed consent was obtained before being included in this study. Thirty age and sex-matched clinically apparently healthy non pregnant females were included as controls. The experimental protocol was approved by the Research Ethical Committee of The Institute of molecular biology and biotechnology, The University of Lahore. Five $\mathrm{ml}$ of cord blood and saliva were taken and centrifuged within one hour of collection and stored at $-70^{\circ} \mathrm{C}$ until assayed. The subjects with the history of taking drugs (including alcohol and cigarette), prediagnosis medications (e.g. antiparkinsonian/antipsychotic), were excluded from this study. None of the controls were on any medication, history of chronic infections, malnutrition syndrome, metabolic dysfunction (Such as diabetes mellitus, liver diseases, cancer) that could interfere with their oxidative metabolites status.

\section{BIOCHEMICAL ANALYSIS}

Lipid peroxidation in sample was estimated by using the method of Ohkawa et al., (1979) [21]. MDA level was assessed by following its possible protocol and absorption was checked with the help of spectrophotometer. The measurements of isoprostanes (IsP) and 8-OHdG (8-
Hydroxyguanosine) were assessed by commercial kits (ENZO) using Eliza. Data was expressed as mean \pm standard deviation. The statistical analysis (Independent t-test) was performed using SPSS version 16. A p value less than 0.05 was taken as statistically significant.

\section{RESULTS}

The present study was carried on 30 subjects and their oxidative stress biomarkers were compared with control. The levels of Malondialdehyde (MDA), Isoprostanes (isop) and 8Hydroxyguanosine ( $8 \mathrm{OHdG}$ ) in saliva, serum and Erythrocytes have been measured. The mean value of MDA (Malondialdehyde) in saliva $(0.48 \pm 0.20 \mathrm{Vs}$ $0.0311 \pm 0.19 \mathrm{nmol} / \mathrm{ml})$, serum $(1.43 \pm 0.48 \mathrm{Vs}$ $1.14 \pm 0.27 \mathrm{nmol} / \mathrm{ml}$ ) and Erythrocytes $(0.34 \pm 0.11 \mathrm{nmol} / \mathrm{ml}$ Vs $0.02 \pm 0.007 \mathrm{nmol} / \mathrm{ml})$ was differed significantly between pregnant and non-pregnant healthy women. Furthermore increased levels of Isoprostanes (Isop) and 8hydroxy guanosine (8OHdg) was observed in saliva $(0.40 \pm 0.29 \mathrm{pg} / \mathrm{ml})$ and $(0.048 \pm 0.02 \quad \mathrm{ng} / \mathrm{ml}), \quad$ serum $(27.90 \pm 1.25 \mathrm{pg} / \mathrm{ml})$ and $(0.32 \pm 0.081 \mathrm{ng} / \mathrm{ml})$ and Erythrocytes $(90.01 \pm 5.36 \mathrm{pg} / \mathrm{ml})$ and $(0.243 \pm 0.103 \mathrm{ng} / \mathrm{ml})$ of subjects as compared to control groups sliva $(0.003 \pm 0.0019 \mathrm{pg} / \mathrm{ml})$ and $\quad(0.0015 \pm \quad 0.00038 \mathrm{ng} / \mathrm{ml}), \quad$ serum $(0.83 \pm 0.57 \mathrm{pg} / \mathrm{ml})$ and $(0.0218 \pm 0.007 \mathrm{ng} / \mathrm{ml})$ 
and Erythrocytes $(1.33 \pm 0.29 \mathrm{pg} / \mathrm{ml})$ and $\quad(0.025 \pm 0.0085 \mathrm{ng} / \mathrm{ml})$ respectively.

Table 1: Prognostic Variables Of Oxidative Stress In Pregnant Womens At The Time Of Delivery

\begin{tabular}{|c|c|c|c|c|c|}
\hline Variables & $\mathbf{n}=\mathbf{3 0}$ & SALIVA & SERUM & ERYTHROCYTES & P-VALUE \\
\hline \multirow{2}{*}{$\begin{array}{c}\text { Malondialdehyde (MDA } \\
\text { nmol/ml) }\end{array}$} & control & $\mathbf{0 . 0 3 1 1} \pm \mathbf{0 . 1 9}$ & $\mathbf{1 . 1 4} \pm \mathbf{0 . 2 7}$ & $\mathbf{0 . 0 2} \pm \mathbf{0 . 0 0 7}$ & $\mathbf{0 . 0 2 3}$ \\
\cline { 2 - 6 } & subjects & $\mathbf{0 . 4 8} \pm \mathbf{0 . 2 0}$ & $\mathbf{1 . 4 3} \pm \mathbf{0 . 4 8}$ & $\mathbf{0 . 3 4} \pm \mathbf{0 . 1 1}$ & $\mathbf{0 . 0 3 8}$ \\
\hline $\begin{array}{c}\text { Isoprostanes } \\
\text { (pg/ml) }\end{array}$ & control & $\mathbf{0 . 0 0 3} \pm \mathbf{0 . 0 0 1 9}$ & $\mathbf{0 . 8 3} \pm \mathbf{0 . 5 7}$ & $\mathbf{1 . 3 3} \pm \mathbf{0 . 2 9}$ & $\mathbf{0 . 0 2 1}$ \\
\cline { 2 - 7 } & subjects & $\mathbf{0 . 4 0} \pm \mathbf{0 . 2 9}$ & $\mathbf{2 7 . 9 0} \pm \mathbf{1 . 2 5}$ & $\mathbf{9 0 . 0 1} \pm \mathbf{5 . 3 6}$ & $\mathbf{0 . 0 0 3}$ \\
\hline $\begin{array}{c}\mathbf{8}-\text { Hydroxy guanosine } \\
\text { (8OHdG)( ng/ml) }\end{array}$ & control & $\mathbf{0 . 0 0 1 5} \pm \mathbf{0 . 0 0 0 3 8}$ & $\mathbf{0 . 0 2 1 8} \pm \mathbf{0 . 0 0 7}$ & $\mathbf{0 . 0 2 5} \pm \mathbf{0 . 0 0 8 5}$ & $\mathbf{0 . 0 1 9}$ \\
\hline & subjects & $\mathbf{0 . 0 4 8} \pm \mathbf{0 . 0 2 1}$ & $\mathbf{0 . 3 2} \pm \mathbf{0 . 0 8 1}$ & $\mathbf{0 . 2 4 3} \pm \mathbf{0 . 1 0 3}$ & $\mathbf{0 . 0 0 6}$ \\
\hline
\end{tabular}

\section{DISCUSSION}

Oxidative state of both mother and baby was assessed soon after delivery, when oxidative state was found higher there will be increased levels of stress markers in umbilical cord blood. This oxidative stress may be same in second and third trimester of pregnancy or may be worsened in third trimester of pregnancy as pregnancy advances, these findings are in agreement to findings that oxidative stress increases during pregnancy by $[22,23,24]$. During pregnancy, human placenta may responsible to induce lipid peroxidation by stimulating increased production of free radicals $[25,16]$. Lipid peroxidation when started in placental trophoblasts and villous core compartment also goes into maternal blood and more lipid peroxidation chain reactions are started [27]. The biomarkers of oxidative stress measured in current study are very important in the growth and development of fetus and are usually related with intrauterine growth retardation $[28,29]$. Although pregnancy is a common physiological condition but it promotes oxidative stress [30, 31]. Antioxidants have defensive function against oxidative stress. Total antioxidant capacity (TAC) represents the balance between oxidative stress and the neutralizing systems. These Oxidative processes are not entirely harmful in pregnancy. First of all they regulate the basic fundamental regulatory functions and in beginning when lipid peroxidation is necessary for trophoblastic inflammatory invasion of maternal tissue, this will arise activation of nitric oxide and free radicals or reactive oxygen species and all these products modulate the metalloproteinases (these metalloproteinases are usually responsible for normal functioning and remolding of uterine extracellular matrix [32]. When there will be imbalance in oxidants and antioxidants the problem occurs which ultimately leads to inadequate reducing capacity of antioxidants [33]. Lipid hydroperoxides function in normal physiology by regulating enzymes 
and redox sensitive genes [34, 35]. This normal physiology is useful in normal healthy pregnancy but problem stared here when this lipid peroxidation becomes uncontrolled and results in cellular damage and there will be increase in vascular resistance (e.g. in gestational disease like preeclampsia). Deficiency of trace elements like selenium and vitamin $\mathrm{E}$ leads to the some functional deficiency of thioredoxin reductase, this is necessary for normal function of RNA and DNA. These deficiencies will also increase oxidative stress of pregnancy that may lead to preeclampsia and eclampsia [36]. Oxidative stress in pregnancy leads to more utilization of antioxidants so this will lead to decrease in total antioxidant capacity in our pregnant ladies. Reduced antioxidants may be a cause of reduced placental efficiency and calcification [37]. It could also be a cause of fetal malformation, pregnancy complications such as preeclampsia and eclampsia as well as aggravate obstructive airway disease and gestational diabetes [38].

Increase production of ROS (Reactive oxygen species), results in increased lipid peroxidation which will in turn damages unsaturated fatty acids mainly localized in cell membrane and DNA in cell which give rise to the formation of isoprostanes (isop),
Malondialdehyde (MDA), and 8 hydroxy guanosine (8OHdg). Isoprostanes and Malondialdehyde are used for lipid peroxidation monitoring in biological samples and 8 hydroxy guanosine are used for measurement of DNA damage in the cells. During the last few years researchers are trying to come up with discovery of new biomarkers which can be helpful in diagnosis and monitoring of prognosis of different oxidative stress induced diseases in newborn and mother during gestation. MDA is one of the most commonly used and reliable biomarker of oxidative stress and due to its toxicity and high reactivity it is very popular to biomedical research community. Malondialdehyde (MDA), interaction with DNA and proteins has often been referred to as potentially mutagenic and atherogenic. Longevity and high reactivity allow the MDA values in uncomplicated pregnancy were higher than in non-pregnant controls. MDA levels were high on this occasion and were reported to be obtained with the method described by [39]. Increase levels of MDA in pre-eclampsia patients as well as the hypothesis of increased oxidative stress in uncomplicated pregnancy were confirmed by [40]. In studies conducted by [41] the mean MDA level in cord blood either delivered vaginally or by c/s 


\section{$(2.03 \pm 0.42 \mathrm{nmol} / \mathrm{ml}, 2.21 \pm 0.95 \mathrm{nmol} / \mathrm{ml}$}

respectively) were significantly higher as compared to control $(0.92 \pm 0.29 \mathrm{nmol} / \mathrm{ml})$. MDA level was significantly higher, in another study conducted by [42] stated that there was increased lipidperoxidation. Our findings also agreed with these studies which indicates the highly significant differential expression of Malondialdehyde in these 3 medias (saliva, serum and erythrocytes) $(0.48 \pm 0.20, \quad 1.43 \pm 0.48,0.34 \pm 0.11 \mathrm{nmol} / \mathrm{ml}$ respectively) as compare to control $(0.0311 \pm 0.019,1.14 \pm 0.27,0.02 \pm 0.007 \mathrm{nmol} / \mathrm{m}$ 1 respectively). This indicates that in pregnancy there are more free radical generation due to high oxygen consumption by fetus and its own body, and it will super imposed by any gestational disease like PIH or gestational diabetes. Findings of current study suggested that MDA measurement plays an important role in the evaluation of oxidative stress in pregnancy.

Second most important key biomarkers of oxidative stress is isoprostanes, also a specific product of lipid peroxidation and reliable biomarkers for investigating the role of free radical generation in pathogenesis of human disorders. Recently various studies have been conducted for quantitative and qualitative analysis of isoprostanes in blood, serum and saliva also in urine. [20]. In present study isoprostanes levels were significantly found to be high in saliva, serum and erythrocytes $(0.40 \pm 0.29, \quad 27.90 \pm 1.25,90.01 \pm 5.36 \mathrm{pg} / \mathrm{ml}$ respectively) as compare to control $90.003 \pm 0.0019,0.83 \pm 0.57,1.33 \pm 0.29 \mathrm{pg} / \mathrm{ml}$ respectively). More significant high level of isoprostanes was observed in erythrocytes as compared to others. Current findings also in accordance with the study of Perrine et al, [43]. 8 hydroxy guanosine is another biomarker used to estimate DNA damage in the cells. In a study conducted by Hasanet al, the mean levels of 8-hydroxy guanosine (a product of DNA damage) were significantly high in inflammatory conditions as well as in normal pregnancy [44]. In current study the levels of 8-hydroxy guanosine was significantly high in saliva, serum and erythrocytes $\quad(0.048 \pm 0.021, \quad 0.32 \pm 0.081$, $0.243 \pm 0.103 \mathrm{ng} / \mathrm{ml}$ respectively) as compare to control $(0.0015 \pm 0.00038,0.0218 \pm 0.007$, $0.025 \pm 0.0085 \mathrm{ng} / \mathrm{ml}$ respectively ) and also more significantly differed in erythrocytes.

\section{CONCLUSION}

It is concluded that MDA level was high in cord blood serum as compared to cord blood erythrocytes and saliva. The highest levels of isoprostanes were observed in cord blood erythrocytes followed by cord blood serum but lowest in saliva. The level of 
8-hydroxyguanosine was also high in cord blood serum but no significant difference with other medias. The levels of isoprostanes in cord blood erythrocytes and cord blood serum have significant diagnostic value and predispositional variables for early detection of oxidative stress generated during normal pregnancy and used for future perinatal outcomes to assess the suspected complications.

\section{ACKNOWLEDGEMENTS}

The authors are highly thankful to the Director IMBB for providing ambient environment to favour research activities and to the students of IMBB Lab-313 for their consistent hardwork.

\section{CONFLICT OF INTEREST}

Authors declare no conflict of interest.

\section{REFERENCES}

[1] Sobolewski K, E Bankowski, L Chyczewski and S Jaworski. Collagen and Glycosaminoglycans of Wharton'S Jelly. Biol Neonate. 1997; 71: 11-21.

[2] Hwai S.W, C.H Shih, T.P Shu, C.H Chun, M.W Hung, J.G Yi, C.C Chin. Mesenchymal Stem Cells in Wharton's Jelly of Human umbilical Cord. Willey online library. 2004; 22: 1330-1337.

[3] Danby R, V Rocha. Improving engrafment and immune reconstitution in umblical cord blood transplantation. Front immunol. 2014; 5: 1-68.

[4] Blau H.M, T.R Brazelton, J.M Weiman. The evolving concept of a stem cell entity or function. Cell. 2001; 105: 829-841.

[5] Santiago R, M.P Jasep, G Calorena, Menton, B.G Antoni. The role and potential of umblical cord blood in an era of new therapies. Stem cell research and therapy. $2015 ; 6: 1-11$.

[6] Jerome L, R.V Nathalie, V Rosenblatt, P Rounten, L Lucas. The role of oxidative stress during inflammatory process. Biol Chem. 2014; 395: 203-230.

[7] Pacher P, J.S Beckman, L Liadet. Nitric oxide and peroxynitrite in health and disease. Physiol. 2007; 87: 315-424.

[8] Devasagayam T.P, K.K Booler, T Ramasarma. Methods for estimating lipid peroxidation: An analysis of merits and demerits. Indian journal of biochemistry. 2003; 40: 300-308.

[9] Daniel A.A, P.O Dolapo, S.A Adeniran, A.A Akinyemi. Effect of different modes of delivery on cord blood oxidative stress markers. International journal of Biomedical Science. 2013; 9(4): 249-254.

[10] Volko M.L, D Monocol, M.T Cronin, M Mazur, J Talser. Free radical and antioxidants in normal physiological functions and human disease. International journal of biochemistry and cell biology. 2007; 39: 44-84. 
[11] Chen A.F, D.D Chen, A Daiber, F Faraci, C.M Rombold, Laher. Free radical biology of the cardiovasculer system. Clinical science. 2012; 123: 73-91.

[12] Sandro A, J.M Maria, A.A Antonio, M lberto, $\mathrm{H}$ Blass. Correlation between circulating biomarkers of oxidative stress of maternal and umblical cord blood at birth. Free Radical Research. 2006; 40(6): 565-570.

[13] Ying,F, Y Tia-Lin. Umblical cord biomarker for pridicting early onset neonatal sepsis. World J Pediatr. 2012; 8(2): 101-108.

[14] Uchenna I.N, E.E Fidelis. Serum ascorbic acid levels during pregnancy in Enugu, Nigeria. J Coll Med. 2005; 10(1): 43-5.

[15] Hracsko Z, H Orvos, Z Novak, A Pal. Evaluation of oxidative stress markers in neonates with intrauterine growth retardation. Redox Rep. 2008; 13(1): 1116.

[16] Mert I, A.S Oruc, S Yuksel. Role of oxidative stress in pre-eclampsia and intrauterine growth restriction. Journal of obstetric and Gyneacology Res. 2012; 38(4): 658-664.

[17] Reena N, P Deepti, K Kanchan, K Ashok, S Ranjana, H.D.Khannal. A novel approach to study oxidative stress in Neonatal respiratory distress syndrome. BBA. Clinical. 2014; 3: 65-69.
[18] Ullas K, R Guruprasad, U.K Shabho, R Lavanya. Maternal and Fetal indicators of oxidative stress during intrauterine growth retardation(IUGR). Indian journal of Clinical biochemistry. 2006; 21(11): 11119.

[19] Morrow J.D, J.A Awad, H.J Boss, I Blair, L.J Roberts. Non-cyclooxugenase derived prostanoids (F2 isoprostanes) are formed in situ on phospholipids. The National Academic of Science -Enginering Medicine.USA. 1992; 89: 10721-10725.

[20] Mario C,S Cinzia, L Silvia, B Giiuseppe, R Viviana, Lucia ciccoli. Plasma F2 isoprostanes are Elevated in Newborns and inversly correlated to gestational age. Free Radical Biology \& Medicine. 2004; 37(5): 724-732.

[21] Ohkawa, H, N Ohishi, K Tagi. Assay for lipid peroxides in animal tissues by thiobarbituric acid reaction. Anal Biochem. 1979; 95:351-358.

[22] Little R.E, B.C Gladen. Levels of lipid peroxides in uncomplicated pregnancy: A review of the literature. Reprod Toxicol. 1999; 13: 347-352.

[23] ToescuV, S.L Nuttali, U Martin, M.J Kendal, F Dunne. Oxidative stress and normal pregnancy. Clinical Endocrinology. 2002; 57: 609-613.

[24] Wang Y.P, S.W Walsh, J.D Guo, J.Y Zhang. Maternal levels of prostacyclin, thromboxane, vitamin E, and lipid 
peroxides throughout normal pregnancy.

Am J Obstet Gynecol. 1991; 165: 16901694.

[25] Winterbourn C.C, T Chan I.H Buss, T.E Inder, N Mogridge B.A Darlow. Protein carbonyls and lipid peroxidation products as oxidation markers in preterm infant plasma: Associations with chronic lung disease and retinopathy and effects of selenium supplementation. Pediatr. 2000; 48: 84-90.

[26] Walsh S.W, Y Wang. Secretion of lipid peroxides by the human placenta. Am J Obstet Gynecol. 1993; 169: 1462-1466.

[27] Walash, Y Wang. Trophoblast and placental villous core production of lipid peroxides, thromboxane, and prosta- cyclin in preeclampsia. J Clin Endocrinol Metab. 1995; 80: 1888-1893.

[28] Karowicz-Bilinska A. Lipid peroxidescon centration in women with intrauterine growth restriction. Ginekol Pol. 2004; 75: 6-9.

[29] Karowicz-Bilinska A, U KowalskaKoprek, J Suzin, P Sieros- zewski. Analysis of 8-isoprostane concentration as a marker of oxidative stress in pregnant women diagnosed with IUGR. Ginekol Pol. 2003; 74: 1137-1142.

[30] Uchenna I.N, E.E Fidelis. Serum ascorbic acid levels during pregnancy in Enugu, Nigeria. J Coll Med. 2005; 10(1): 43-5.
[31] Jonathan M.M, K.G, Nitin, J.R Marit, M Knight, A.L Eliza- beth, D Sumwan. Circulating markers of oxidative stress is raised in normal pregnancy and preeclampsia. Briti J Obs Gyn. 1998; 105: 1195-9.

[32] Biondi C, B Paran, L Langhi, S Fiorni, F Vesce. The role and Modulation of Oxidative balance in pregnancy. Current pharmaceutical Design. 2005; 11(16): 2075-2089.

[33] Stark J.M. Inadequate reducing system in pre-eclamp- sia: a complementary role for vitamin $\mathrm{C}$ and $\mathrm{E}$ with thioredoxin-related activities. Briti J Obs Gyn. 2001; 108: 33943.

[34] Smith M.E, M.K V ander, F.P Somera. Macrophage and macroglia responses to cytokines in vitro:phagocytic activity,proteolytic enzyme release and Radical production.The Journal of Neuroscience Research. 1998; 54: 68-78.

[35] Sen C.K, L Packer. Antioxidant and redox regulation of gene transcription. FASEB J. 1996; 10: 709-20.

[36] Stark J.M. Inadequate reducing system in pre-eclamp- sia: a complementary role for vitamin $\mathrm{C}$ and $\mathrm{E}$ with thioredoxin-related activities. Briti J Obs Gyn. 2001; 108: 33943.

[37] Klesgee L.M, D.M Murray, J.E Brow, S.P Cliver, R.L Goldenberg. Relations of cigarette smoking and dietary antioxidants 
with placenta calcification. Amer $\mathrm{J}$ Epidemiology. 1998; 147: 127-35.

[38] Hug Cassino P.A. Antioxidant nutrients and pulmo- nary function: The third National and Nutrition Examination Survey (NHAMES III). Am J Epidol. 2000; 151(10): 975-81.

[39] Richard M.J, J Arnaud, C Jurkovitz, T Hachache, H Meftahi, Laporte. Trace elements and lipid peroxidation abnormalities in patients with chronic renal failure. Nephron. 1991; 57: 10-5.

[40] Ilhan N, M Simsek. The changes of trace elements, malondialdehyde levels and superoxide dismutase activi- ties in pregnancy with or without preeclampsia. Clin Biochem. 2002; 35: 393-97.
[41] Sayat G.M.D, A Vefik, H Sami, K Ayse, A Seci, K Mand Guner. Malondialdehyde level in cord blood of newborn infants. The journal of pediatrics. 2011; 21(3): 313-319.

[42] Bilgili G, C Dizdarer, S Kalkan. Relationship between the factors in perinatal period and Malondialdehyde concentration in cord blood. Ege J Pediatr. 2005; 12(1): 7-12.

[43] Perrone S, R Bracci, G Buonocore. New biomarkers of Fetal-Neonatal hypoxic stress. Acta Paediatrica. 2002;438:135-138.

[44] Hasan, M, Mohieldein, A. H., Almutairi, F. R. Comparative study of serum 8hydroxydeoxy-guanosine levels among healthy offspring of diabetic and nondiabetic parents. International journal of health sciences. 2017; 11(3): 33 . 